\title{
Berechnung des Dentalmikroskopes in der Endodontie beim PKV- und GKV-Patienten
}

\author{
Im Bewertungsmaßstab zahnärztlicher Leistungen \\ (BEMA) existiert keine Gebührennummer für die \\ Anwendung des Dentalmikroskopes. Nachdem im \\ Unterschied zur Gebührenordnung für Ärzte (GOÄ) \\ das Operationsmikroskop auch in der privaten \\ Zahnheilkunde gebührentechnisch lange nicht be- \\ rücksichtigt worden ist, hat der Verordnungsgeber \\ bei der Reform der Gebührenordnung für Zahnärzte \\ (GOZ) 2012 versucht, den Aufwand für die Anwen- \\ dung eines Dentalmikroskopes in der Zahnheilkun- \\ de abzubilden.
}

Dieser Versuch hat allerdings keine positiven Auswirkungen. Herausgekommen ist dabei die nicht steigerungsfähige $\mathrm{Zu}$ schlagsposition GOZ-Nr. 0110: „Zuschlag für die Anwendung eines Operationsmikroskops bei den Leistungen nach den Nummern 2195, 2330, 2340, 2360, 2410, 2440, 3020, 3030, 3040, $3045,3060,3110,3120,3190,3200,4090,4100,4130,4133,9100$, 9110, 9120, 9130 und 9170." Für die Anwendung gibt es pauschal 22,50 Euro. Die GOZ-Nr. 0110 gibt damit vor, dass sie nur bei diesen ganz klar definierten aufgelisteten Gebührennummern der GOZ berechnet werden kann und sonst nirgends.

\section{Zuzahlungen sind nicht erlaubt}

Eine Vereinbarung der GOZ-Nr. 0110 ist mit gesetzlich versicherten Patienten daher neben einer Sachleistung (zum Beispiel einer Wurzelkanalaufbereitung oder -füllung) aus dem BEMA nicht möglich. Auch wenn es zur Qualitätsverbesserung und zur Verbreitung von Dentalmikroskopen sehr wünschenswert wäre, beispielsweise neben der BEMA-Nr. 32 (Wurzelkanalaufbereitung), 35 (Wurzelkanalfüllung) oder 54 (Wurzelspitzenresektion) einen Zuschlag für die Anwendung des Dentalmikroskopes anzusetzen, so ist dies gebührenrechtlich nicht möglich, weder durch einen Ansatz der GOZ-Nr. 0110 noch durch eine andere GOZ-Position. Zuzahlungen zu den vertragszahnärztlichen (hier endodontischen) Leistungen sind nach den Vorgaben der Bundesmantelverträge nicht erlaubt.

Was also tun, wenn man sich sicher ist, dass die Hinzuziehung eines Dentalmikroskopes bei einer geplanten Wurzelbehandlung zu besseren Ergebnissen führen würde und man dem Patienten diese Entwicklung der Zahnheilkunde nicht vorenthalten will? Sollte der Zahnarzt feststellen, dass ohne Hinzuziehung eines Dentalmikroskopes die Prognose des zu behandelnden Zahnes infaust oder derart fragwürdig ist, dass es nicht den Behandlungsrichtlinien des BEMA entspräche, diesen Zahn im Rahmen der gesetzlichen Krankenversicherung endodontisch zu behandeln, so ist dieser Zahn vollständig privat zu therapieren (siehe auch „Endo-Richtlinien“ der vertragszahnärztlichen Behandlung, Ziffer B III.9. und auch 10).

Dies bedeutet dann also, dass die gesamte Wurzelbehandlung nach den privaten GOZ-Gebührennummern 2390-2440 erfol- gen muss. Dann kann auch der Zuschlag GOZ-Nr. 0120 (aber nur einmal je Sitzung bzw. „Behandlungstag“) zu den GOZNummern 2360 oder 2410 oder 2440 erfolgen.

Eine Zuordnung des Dentalmikroskop-Zuschlages GOZ-Nr. 0110 zu Maßnahmen, die zur „GKV-Endo“ gesondert privat vereinbart wurden und im BEMA nicht enthalten sind, wie die elektrometrische Längenmessung (GOZ-Nr. 2400) oder elektrophysikalisch-chemische Anwendungen (GOZ-Nr. 2420), ist ebenfalls nicht möglich, da eben auch diese beiden Nummern 2400 und 2420 entsprechend den Vorgaben der Leistungslegende nicht mit dem Zuschlag 0110 kombiniert werden dürfen.

\section{Honoraranpassung an den Aufwand}

Werden die erwähnten 22,50 Euro für die GOZ-Nr. 0110 dem tatsächlichen Aufwand beim Einsatz des Dentalmikroskopes nicht gerecht, so besteht nach Auffassung der Bundeszahnärztekammer die Möglichkeit, eine Honorarvereinbarung nach $\$ 2$ Abs. 1 und 2 auch über die Zuschlagsnummer 0110 zu treffen. Hierdurch wird es möglich, auch einen sehr zeitintensiven und sehr hochwertigen Einsatz des Dentalmikroskopes in der Honorarhöhe passend abzubilden.

Wird ein Dentalmikroskop angewendet bei endodontischen oder anderweitigen Privatbehandlungen im Zusammenhang mit Leistungen, die nicht in der Leistungslegende der GOZ-Nr. 0110 aufgelistet sind, so stellen die besonderen Umstände und der höhere Zeitaufwand durch das Dentalmikroskop einen guten Grund für einen höheren Steigerungsfaktor nach $₫ 5,2 \mathrm{GOZ}$ oder auch für eine Honorarvereinbarung nach $₫ 2$ Abs. 1 und 2 bei der Berechnung der jeweiligen Privatleistung dar.

Besonders beachtet werden sollte auch, dass bestimmte neuartige diagnostische Leistungen, wie beispielsweise das Aufsuchen von intrakanalären Rissen, was mithilfe von Farbstoffen und Dentalmikroskopen erst ermöglicht wird, zum Beispiel nach Auffassung der Landeszahnärztekammer Baden-Württemberg eine selbstständige Leistung darstellen, die dann insgesamt analog nach $₫ 6,1 \mathrm{GOZ}$ berechnet wird. Dies gilt für privat versicherte Patienten ebenso wie für GKV-Patienten, bei denen diese Leistung natürlich - wie immer - zuvor privat vereinbart werden muss.

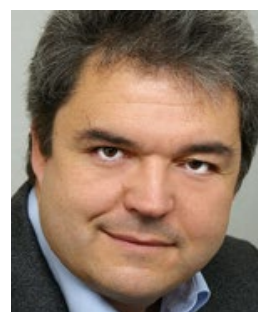

Dr. Dr. Alexander Raff

Mitglied im GOZ-Expertenrat des FVDZ 